\title{
A design procedure for overlapped guaranteed cost controllers
}

\author{
Francisco Palacios *, José Rodellar** and Josep M. Rossell * \\ * Department of Applied Mathematics III, Universitat Politècnica de \\ Catalunya (UPC), Campus Manresa, 08242, Manresa, Spain (e-mail: \\ francisco.palacios@upc.edu),(e-mail: josep.maria.rossell@upc.edu) \\ ** Department of Applied Mathematics III, Universitat Politècnica de \\ Catalunya (UPC), Campus Nord, C-2, 08034, Barcelona, Spain (e-mail: \\ jose.rodellar@upc.edu).
}

\begin{abstract}
In this paper a quadratic guaranteed cost control problem for a class of linear continuous-time state-delay systems with norm-bounded uncertainties is considered. We will suppose that the systems are composed by two overlapped subsystems but the results can be easily extended to any number of subsystems. The main objective is to design overlapping guaranteed cost controllers with tridiagonal gain matrices for these kind of systems by using a linear matrix inequality (LMI) approach. With this idea in mind, we present a design strategy to reduce the computational burden and to increase the feasibility in the LMI problem. In this context, the use of so-called complementary matrices play an important role. A simple example to illustrate the advantages achieved by using the proposed method is supplied.
\end{abstract}

Keywords: Decentralized control, overlapping decomposition, inclusion principle, guaranteed cost, linear matrix inequalities (LMIs)

\section{INTRODUCTION}

Many physical systems are described by time-delay model uncertainties. Time-delay is a source of instability and systems having this characteristic have been widely studied in control systems literature. Delays can appear, for instance, in the transmission of information between different parts of a system: communication systems, chemical processing systems or power systems are some examples.

In presence of uncertainties, time-delay is particularly relevant. Therefore, the design of robust controllers becomes necessary. The basic idea is to design control laws which make the resulting closed-loop systems not only asymptotically stable but also guaranteeing an adequate level of performance. The performance is measured with the standard quadratic cost function and an upper bound for the cost function is defined. An LMI approach will be used to obtain control laws.

In some applications it is required to have gain matrices with a given structure to reduce the cost of information exchange, keeping a level of performance. Adding these kind of restrictions to the matrix variables involved in LMIs, based control design may increase the computational effort or even lead to infeasible LMIs. An alternative way can be given by the Inclusion Principle, Ikeda and Šiljak [1980], Ikeda and Šiljak [1986], Ikeda et al. [1981], Ikeda et al. [1984a], Ikeda et al. [1984b], Šiljak [1991].

Basically, this principle offers the conditions under which a given system, with shared components, can be expanded to a higher dimensional space so that the overlapped subsystems appear as disjoint. The expanded space contains the essential

\footnotetext{
* This work was supported in part by the Committee for Science and Technology (CICYT) of Spain under Grant DPI2005-08668-C03-01
}

information about the initial one in such a manner that a control methodology can be advantageously designed for this system and transformed (contracted) to have a final control law which is implementable into the original system.

The initial and expanded systems are related by appropriate linear transformations. These transformations involve a set of socalled complementary matrices. The selection of these matrices produces expanded systems satisfying different requirements. The influence of these matrices has been illustrated in previous works, Bakule et al. [2000b], Bakule et al. [2000a], Bakule et al. [2001a], Bakule et al. [2002a], Bakule et al. [2001b].

Linear quadratic control has been the methodology mostly considered in this framework, Ikeda et al. [1981], Bakule et al. [2000b], Bakule et al. [2002a]. Other control methods have been adopted together with overlapping decomposition, mainly to cope with uncertainties, like guaranteed cost control, Bakule et al. [2002b], Bakule et al. [2005b] or $H_{\infty}$ control, Bakule et al. [2005c], Li et al. [2000]. The inclusion principle has been studied and applied satisfactory in different areas as mechanical systems, Bakule and Rodellar [1995], electric power systems Stanković et al. [1999], vehicles Stanković et al. [2000], Stipanović et al. [2004], control of structures, Bakule et al. [2005a] or applied mathematics, Sezer and Šiljak [1991].

Frequently, it is convenient to utilize structures which offer maximal improvement in performance at a minimal cost in information exchange, Šiljak and Zečević [2005]. Treating with overlapping decompositions, we are interested in designing controllers so that the corresponding gain matrices have a tridiagonal form. To do this, a given system is expanded to a larger dimensional one. In this process the role of the complementary matrices is crucial to obtain a new system decomposed into subsystems with null or weak interconnections. For each single 
subsystem an LMI approach is used to design a local guaranteed cost control, Mukaidani [2003]. Under the scope of the inclusion principle, the overall diagonal gain matrix is contracted to an implementable control in the original system. The obtained controller will have the required tridiagonal form, guaranteeing stability and an upper cost bound.

We want to note that it is possible to design these gain matrices directly in the initial system. However, to do this it is necessary to impose restricted structural conditions on the LMIs. At the same time, working with large-scale systems the computational cost can be very high. Thus, the design procedure proposed in this paper may have two main advantages: (1) the reduction of computational complexity because LMIs are applied to smaller dimensional decoupled subsystems instead of an overall full system; (2) structural restrictions are not imposed to the unknown matrices in the LMIs, with subsequent increase in its feasibility as compared with the restricted centralized case.

The paper is organized as follows. Section II presents necessary background results. Section III offers a design procedure to solve the problem, while Section IV provides an illustrative numerical example. The final conclusions are given in Section $\mathrm{V}$.

\section{PRELIMINARIES}

Consider a pair of linear continuous-time uncertain systems with state delay described by the state equations

$$
\begin{aligned}
\mathbf{S}: \dot{x}(t) & =[A+\Delta A(t)] x(t)+[B+\Delta B(t)] u(t) \\
& +[C+\Delta C(t)] x(t-d), \\
x(t) & =\varphi(t), \quad-d \leq t \leq 0, \\
\tilde{\mathbf{S}}: \quad \dot{\tilde{x}}(t) & =[\tilde{A}+\Delta \tilde{A}(t)] \tilde{x}(t)+[\tilde{B}+\Delta \tilde{B}(t)] u(t) \\
& +[\tilde{C}+\Delta \tilde{C}(t)] \tilde{x}(t-d), \\
\tilde{x}(t) & =\tilde{\varphi}(t), \quad-d \leq t \leq 0,
\end{aligned}
$$

where $x(t) \in \mathbb{R}^{\mathbf{n}}$ and $u(t) \in \mathbb{R}^{\mathbf{m}}$ are the state and the input of $\mathbf{S}$, $\tilde{x}(t) \in \mathbb{R}^{\tilde{\mathbf{n}}}$ and $u(t) \in \mathbb{R}^{\mathbf{m}}$ are the corresponding to $\tilde{\mathbf{S}}$. Denote $\varphi(t)$ a continuous vector valued initial function. Denote $x_{0}=x(0)$ and $\tilde{x}_{0}=\tilde{x}(0)$ the initial states of the systems $\mathbf{S}$ and $\tilde{\mathbf{S}}$, respectively. The sets $\{x(t), u(s)\}$ and $\{\tilde{x}(t), u(s)\}$ with $s \in[t-d, t]$ define complete states for (1) and (2), respectively. Suppose that the dimension of the state vector $x(t)$ of $\mathbf{S}$ is smaller than the vector $\tilde{x}(t)$ of $\tilde{\mathbf{S}}$. The matrices $A, B, C, \tilde{A}, \tilde{B}$ and $\tilde{C}$ are constant of appropriate dimensions. $\Delta A(t), \Delta B(t), \Delta C(t), \Delta \tilde{A}(t), \Delta \tilde{B}(t)$ and $\Delta \tilde{C}(t)$ are real-valued matrices of uncertain parameters. Uncertainties are assumed to be norm-bounded as follows:

$$
\begin{aligned}
{[\Delta A(t) \Delta B(t) \Delta C(t)] } & =E F(t)\left[\begin{array}{lll}
E_{1} & E_{2} & E_{3}
\end{array}\right], \\
{[\Delta \tilde{A}(t) \Delta \tilde{B}(t) \Delta \tilde{C}(t)] } & =\tilde{E} \tilde{F}(t)\left[\begin{array}{lll}
\tilde{E}_{1} & \tilde{E}_{2} & \tilde{E}_{3}
\end{array}\right],
\end{aligned}
$$

where $E, E_{1}, E_{2}, E_{3}, \tilde{E}, \tilde{E}_{1}, \tilde{E}_{2}$ and $\tilde{E}_{3}$ are known constant real matrices of appropriate dimensions and $F(t), \tilde{F}(t)$ are unknown matrix functions with Lebesgue measurable elements such that

$$
F^{T}(t) F(t) \leq I, \quad \tilde{F}^{T}(t) \tilde{F}(t) \leq I .
$$

Associated with the systems $\mathbf{S}$ and $\tilde{\mathbf{S}}$ we have the following cost functions:

$$
\begin{aligned}
& J\left(x_{0}, u\right)=\int_{0}^{\infty}\left[x^{T}(t) Q^{*} x(t)+u^{T}(t) R^{*} u(t)\right] d t, \\
& \tilde{J}\left(\tilde{x}_{0}, u\right)=\int_{0}^{\infty}\left[\tilde{x}^{T}(t) \tilde{Q}^{*} \tilde{x}(t)+u^{T}(t) \tilde{R}^{*} u(t)\right] d t,
\end{aligned}
$$

respectively. $Q^{*}, \tilde{Q}^{*}$ are symmetric positive semi-definite matrices and $R^{*}, \tilde{R}^{*}$ are symmetric positive-definite matrices.
In order to simplify the notation, denote:

$$
\bar{A}(t)=A+\Delta A(t), \quad \bar{B}(t)=B+\Delta B(t), \quad \bar{C}(t)=C+\Delta C(t)
$$

and

$$
\overline{\tilde{A}}(t)=\tilde{A}+\Delta \tilde{A}(t), \quad \overline{\tilde{B}}(t)=\tilde{B}+\Delta \tilde{B}(t), \quad \overline{\tilde{C}}(t)=C+\Delta \tilde{C}(t) .
$$

Then, the systems (1) and (2) can be rewritten as:

$$
\begin{aligned}
\mathbf{S}: \dot{x}(t) & =\bar{A}(t) x(t)+\bar{B}(t) u(t)+\bar{C}(t) x(t-d), \\
x(t) & =\varphi(t), \quad-d \leq t \leq 0, \\
\tilde{\mathbf{S}}: \dot{\tilde{x}}(t) & =\overline{\tilde{A}}(t) \tilde{x}(t)+\overline{\tilde{B}}(t) u(t)+\overline{\tilde{C}}(t) x(t-d), \\
\tilde{x}(t) & =\tilde{\varphi}(t), \quad-d \leq t \leq 0,
\end{aligned}
$$

respectively. Having initial complete states $\left\{x_{0}, u(s)\right\}$ and $\left\{\tilde{x}_{0}, u(s)\right\}$ for the systems $\mathbf{S}$ and $\tilde{\mathbf{S}}$, the unique solutions of (10) and (11) are given by

$$
\begin{aligned}
x(t)=x\left(t ; x_{0}, u\right) & =\Phi(t, 0) x(0)+\int_{0}^{t} \Phi(t, s) \bar{C}(s) x(s-d) d s \\
& +\int_{0}^{t} \Phi(t, s) \bar{B}(s) u(s) d s
\end{aligned}
$$

and

$$
\begin{aligned}
\tilde{x}(t)=\tilde{x}\left(t ; \tilde{x}_{0}, u\right) & =\tilde{\Phi}(t, 0) \tilde{x}(0)+\int_{0}^{t} \tilde{\Phi}(t, s) \tilde{\bar{C}}(s) \tilde{x}(s-d) d s \\
& +\int_{0}^{t} \tilde{\Phi}(t, s) \tilde{\bar{B}}(s) u(s) d s,
\end{aligned}
$$

respectively, where $\Phi$ and $\tilde{\Phi}$ are the transition matrices corresponding to $\bar{A}(t)$ and $\overline{\tilde{A}}(t)$.

Remark. To simplify the problem, only state expansion is considered in the paper. However, a similar process can be made when the control vector is expanded too.

\subsection{An LMI Approach}

In the literature, there are available different approaches to compute quadratic guaranteed cost controllers. In this paper, a delay LMI approach is selected to design a linear state memoryless feedback controller guaranteeing that the system is quadratically stable with a desired upper bound on the quadratic cost function.

Definition 1. A control law $u(t)=K x(t)$ is said to define a quadratic guaranteed cost control with associated cost matrix $P>0$ for the delay system (1) with a cost function (6) if the corresponding closed-loop system is asymptotically stable for all nonzero $x \in \mathbb{R}^{n}$ and the closed-loop value of the cost function (6) satisfies the bound $J\left(x_{0}, u\right) \leq J^{*}$ for all admissible uncertainties (3).

An analogous definition can be given for a quadratic guaranteed cost control for the system $\tilde{\mathbf{S}}$.

The following proposition gives sufficient conditions to get a guaranteed cost control, Mukaidani [2003]. To simplify, the result is presented only for the system (1) satisfying (3) and (5) together with a cost function given in (6), but it evidently can be stated for the system $\tilde{\mathbf{S}}$ given in (2).

Theorem 2. Suppose there exist constant parameters $\mu>0, \varepsilon>0$, symmetric positive-definite matrices $X, S, Z \in \mathbb{R}^{n \times n}$ and a matrix $Y \in \mathbb{R}^{m \times n}$ such that the following LMI 


$\left[\begin{array}{ccccccc}\Psi & {\left[E_{1} X+E_{2} Y\right]^{T}} & X & Y^{T} & C S & 0 & X \\ E_{1} X+E_{2} Y & -\mu I & 0 & 0 & 0 & 0 & 0 \\ X & 0 & -\left[Q^{*}\right]^{-1} & 0 & 0 & 0 & 0 \\ Y & 0 & 0 & -\left[R^{*}\right]^{-1} & 0 & 0 & 0 \\ S C^{T} & 0 & 0 & 0 & -S & S E_{3}^{T} & 0 \\ 0 & 0 & 0 & 0 & E_{3} S & -\varepsilon I & 0 \\ X & 0 & 0 & 0 & 0 & 0 & -S\end{array}\right]<0$

is feasible, where $\Psi:=A X+B Y+(A X+B Y)^{T}+Z+(\mu+\varepsilon) E E^{T}$. Then, the feedback control law $u(t)=K x(t)=Y X^{-1} x(t)$ is a quadratic guaranteed cost controller for the closed-loop uncertain time-delay system and satisfies

$$
J\left(x_{0}, u\right) \leq J^{*}=\varphi^{T}(0) X^{-1} \varphi(0)+\int_{-d}^{0} \varphi^{T}(s)\left[S^{-1}+X^{-1} Z X^{-1}\right] \varphi(s) d s .
$$

\subsection{Inclusion Principle}

Let us consider the following transformations:

$$
V: \mathbb{R}^{\mathbf{n}} \longrightarrow \mathbb{R}^{\tilde{\mathbf{n}}}, \quad U: \mathbb{R}^{\tilde{\mathbf{n}}} \longrightarrow \mathbb{R}^{\mathbf{n}},
$$

where $V$ and $U$ are full-rank matrices such that $U V=I$. The matrix $V$ is a pseudoinverse of $U$ and can be obtained as $U=\left(V^{T} V\right)^{-1} V^{T}$.

Definition 3. (Inclusion Principle) A system $\tilde{\mathbf{S}}$ includes the system $\mathbf{S}$, denoted by $\tilde{\mathbf{S}} \supset \mathbf{S}$, if there exists a pair of matrices $(U, V)$ satisfying $U V=I$ and such that for any initial state $x_{0}$ and any fixed input $u(t)$ of $\mathbf{S}$, the choice $\tilde{x}_{0}=V x_{0}$ of the system $\tilde{\mathbf{S}}$ implies $x\left(t ; x_{0}, u\right)=U \tilde{x}\left(t ; V x_{0}, u\right)$ for all $t$. If $\tilde{\mathbf{S}} \supset \mathbf{S}$, then $\tilde{\mathbf{S}}$ is said to be an expansion of $\mathbf{S}$ and $\mathbf{S}$ is a contraction of $\tilde{\mathbf{S}}$.

There are two particular but important cases within the inclusion principle: restrictions and aggregations.

Definition 4. A system $\mathbf{S}$ is a restriction of $\tilde{\mathbf{S}}$, if there exists a pair of matrices $(U, V)$ satisfying $U V=I$ and such that for any initial state $x_{0}$ and any fixed input $u(t)$ of $\mathbf{S}$, the choice $\tilde{x}_{0}=V x_{0}$ implies $\tilde{x}\left(t ; \tilde{x}_{0}, u\right)=V x\left(t ; x_{0}, u\right)$ for all $t$.

Definition 5. A system $\mathbf{S}$ is an aggregation of $\tilde{\mathbf{S}}$ if there exists a pair of matrices $(U, V)$ satisfying $U V=I$ and such that for any initial state $\tilde{x}_{0}$ and any fixed input $u(t)$ of $\tilde{\mathbf{S}}$, the choice $x_{0}=U \tilde{x}_{0}$ implies $x\left(t ; x_{0}, u\right)=U \tilde{x}\left(t ; \tilde{x}_{0}, u\right)$ for all $t$.

Definition 6. A control law $u(t)=\tilde{K} \tilde{x}(t)$ designed in the system $\tilde{\mathbf{S}}$ is contractible to $u(t)=K x(t)$ of $\mathbf{S}$ if the choice $\tilde{\varphi}(t)=V \varphi(t)$ implies $K x(t ; \varphi(t), u(t))=\tilde{K} \tilde{x}(t ; V \varphi(t), u(t))$ for all $t$, any initial function $\varphi(t)$ and any fixed input $u(t)$.

Suppose given a pair of matrices $(U, V)$. Then, the matrices $\tilde{A}$, $\Delta \tilde{A}, \tilde{B}, \Delta \tilde{B}, \tilde{C}, \Delta \tilde{C}, \tilde{Q}^{*}$ and $\tilde{R}^{*}$ can be described as:

$$
\begin{aligned}
\tilde{A} & =V A U+M, & \Delta \tilde{A}(t) & =V \Delta A(t) U, \\
\tilde{B} & =V B+N, & \Delta \tilde{B}(t) & =V \Delta B(t), \\
\tilde{C} & =V C U+M_{d}, & \Delta \tilde{C}(t) & =V \Delta C(t) U, \\
\tilde{Q}^{*} & =U^{T} Q^{*} U+M_{Q^{*}}, & \tilde{R}^{*} & =R^{*}+N_{R^{*}},
\end{aligned}
$$

where $M, N, M_{d}, M_{Q^{*}}$ and $N_{R^{*}}$ are so-called complementary matrices. Usually, the transformations $(U, V)$ are selected a priori to define structural relations between the state variables in both systems $\mathbf{S}$ and $\tilde{\mathbf{S}}$. Given these transformations, the choice of the complementary matrices provides degrees of freedom to obtain different expanded spaces with desirable properties, Bakule et al. [2000b], Bakule et al. [2000a]. For $\tilde{\mathbf{S}}$ to be an expansion of $\mathbf{S}$, a proper selection of $M$ and $N$ is required, Ikeda and Šiljak [1980], Ikeda and Šiljak [1986], Ikeda et al. [1981], Ikeda et al. [1984a], Šiljak [1991].
In this paper we assume that the structure of the matrices $A, B$ and $C$ given in (1) have the form:

$$
A, C=\left[\begin{array}{cc:c}
*_{11} & *_{12} & *_{13} \\
*_{21} & *_{22}^{*} & *_{23} \\
\hdashline *_{31} & *_{32} & *_{33}
\end{array}\right], \quad B=\left[\begin{array}{ll}
B_{11} & B_{12} \\
B_{21} & B_{22} \\
B_{31} & B_{32}
\end{array}\right],
$$

where the submatrices $(*)_{i i}$ and $B_{i j}$ for $i=1,2,3, j=1,2$ are $n_{i} \times n_{i}$ and $n_{i} \times m_{j}$ dimensional matrices, respectively. The matrices $A$ and $C$ of $\mathbf{S}$ are composed by subsystems with one overlapped part corresponding to $A_{22}$ and $C_{22}$. This structure has been extensively adopted as prototype in the literature in the context of the inclusion principle.

Considering the overlapping structure (18) in the original system, a standard particular selection of the transformation matrix $V$ is given by

$$
V=\left[\begin{array}{ccc}
I_{n_{1}} & 0 & 0 \\
0 & I_{n_{2}} & 0 \\
0 & I_{n_{2}} & 0 \\
0 & 0 & I_{n_{3}}
\end{array}\right]
$$

This transformation leads in a simple natural way to an expanded system where the state vector $x_{2}(t)$ appears repeated in $\tilde{x}(t)^{T}=\left(\tilde{x}_{1}^{T}(t), \tilde{x}_{2}^{T}(t)\right)=\left(x_{1}^{T}(t), x_{2}^{T}(t), x_{2}^{T}(t), x_{3}^{T}(t)\right)$.

The proofs of the following theorems and propositions can be found in Bakule et al. [2005b].

Theorem 7. Consider the systems (1) and (2) verifying (3), (4) and (5). Then $\tilde{\mathbf{S}} \supset \mathbf{S}$ if and only if

$$
\begin{aligned}
& U \tilde{\Phi}(t, 0) V=\Phi(t, 0), \quad U \tilde{\Phi}(t, s) M_{d} V=0, \\
& U \tilde{\Phi}(t, s) N=0
\end{aligned}
$$

for all $t$ and $s$.

Next, we summarize the main results about the inclusion principle in terms of complementary matrices. Thus, the Theorem 7 can be rewritten in the following form.

Theorem 8. Consider the systems (1) and (2) verifying (3), (4) and (5). Then $\tilde{\mathbf{S}} \supset \mathbf{S}$ if and only if

$$
U M^{i} V=0, \quad U M^{i-1} M_{d} V=0, \quad U M^{i-1} N=0
$$

for all $i=1,2, \ldots, \tilde{n}$.

Proposition 9. Consider the systems (1) and (2) verifying (3), (4) and (5). A system $\mathbf{S}$ is a restriction of the system $\tilde{\mathbf{S}}$ if and only if $M V=0, M_{d} V=0$ and $N=0$.

Proposition 10. Consider the systems (1) and (2) verifying (3), (4) and (5). A system $\mathbf{S}$ is an aggregation of the system $\tilde{\mathbf{S}}$ if and only if $U M=0, U M_{d}=0$ and $U N=0$.

Theorem 11. Consider the systems (1) and (2) verifying (3), (4) and (5) with the structures given in (18) and (19). Suppose that $M_{d}=0$. Then, $\tilde{\mathbf{S}} \supset \mathbf{S}$ if and only if the complementary matrices $M$ and $N$ have the following form:

$$
M=\left[\begin{array}{cccc}
0 & M_{12} & -M_{12} & 0 \\
M_{21} & M_{22} & M_{23} & M_{24} \\
-M_{21} & -\left(M_{22}+M_{23}+M_{33}\right) & M_{33} & -M_{24} \\
0 & M_{42} & -M_{42} & 0
\end{array}\right], N=\left[\begin{array}{cc}
0 & 0 \\
N_{21} & N_{22} \\
-N_{21} & -N_{22} \\
0 & 0
\end{array}\right]
$$

and verify the conditions

$$
\begin{aligned}
& {\left[\begin{array}{c}
M_{12} \\
M_{23}+M_{33} \\
M_{42}
\end{array}\right]\left[M_{22}+M_{33}\right]^{i-1}\left[\begin{array}{lll}
M_{21} & M_{22}+M_{23} & M_{24}
\end{array}\right]=0,} \\
& {\left[\begin{array}{c}
M_{12} \\
M_{23}+M_{33} \\
M_{42}
\end{array}\right]\left[M_{22}+M_{33}\right]^{i-1}\left[\begin{array}{ll}
N_{21} & N_{22}
\end{array}\right]=0}
\end{aligned}
$$

for all $i=1,2, \cdots, \tilde{n}-1$. 
By assuming that $M_{d}=0$, we have the next two propositions.

Proposition 12. A system $\mathbf{S}$ is a restriction of the system $\tilde{\mathbf{S}}$ if and only if the matrices $M$ and $N$ have the following structures:

$$
M=\left[\begin{array}{llll}
0 & M_{12} & -M_{12} & 0 \\
0 & M_{22} & -M_{22} & 0 \\
0 & M_{32} & -M_{32} & 0 \\
0 & M_{42} & -M_{42} & 0
\end{array}\right], \quad N=\left[\begin{array}{ll}
0 & 0 \\
0 & 0 \\
0 & 0 \\
0 & 0
\end{array}\right] \text {. }
$$

Proposition 13. A system $\mathbf{S}$ is an aggregation of the system $\tilde{\mathbf{S}}$ if and only if the matrices $M$ and $N$ have the following structures:

$$
M=\left[\begin{array}{cccc}
0 & 0 & 0 & 0 \\
M_{21} & M_{22} & M_{23} & M_{24} \\
-M_{21} & -M_{22} & -M_{23} & -M_{24} \\
0 & 0 & 0 & 0
\end{array}\right], \quad N=\left[\begin{array}{cc}
0 & 0 \\
N_{21} & N_{22} \\
-N_{21} & -N_{22} \\
0 & 0
\end{array}\right] \text {. }
$$

Remark. By using the transformation $V$ given in (19), Theorem 11 provides the most general structure and the conditions on the complementary matrices $M$ and $N$ so that $\tilde{\mathbf{S}} \supset \mathbf{S}$, assuming that (23) is satisfied. Obviously, the matrices $M$ and $N$ given by Propositions 12 and 13 satisfy (23).

\subsection{Expansion-decoupling-contraction process}

Let $\tilde{\mathbf{S}}$ be the system given in (11) with the structure (18) which is expanded by means of the matrix $V$ given by (19) in the form

$$
\begin{aligned}
\tilde{\mathbf{S}}: \dot{\tilde{x}}(t) & =\overline{\tilde{A}}(t) \tilde{x}(t)+\overline{\tilde{B}}(t) u(t)+\overline{\tilde{C}}(t) x(t-d), \\
\tilde{x}(t) & =\tilde{\varphi}(t), \quad-d \leq t \leq 0,
\end{aligned}
$$

where $\dot{\tilde{x}}(t)=\left[\begin{array}{c}\dot{\tilde{x}}_{1}(t) \\ \tilde{\tilde{x}}_{2}(t)\end{array}\right], \quad \overline{\tilde{A}}(t)=\left[\begin{array}{cc}\overline{\tilde{A}}_{11}(t) & \overline{\tilde{A}}_{12}(t) \\ \tilde{\tilde{A}}_{21}(t) & \tilde{\tilde{A}}_{22}(t)\end{array}\right], \quad \overline{\tilde{B}}(t)=\left[\begin{array}{cc}\overline{\tilde{B}}_{11}(t) & \overline{\tilde{B}}_{12}(t) \\ \tilde{\tilde{B}}_{21}(t) & \tilde{\tilde{B}}_{22}(t)\end{array}\right]$, $\overline{\tilde{C}}(t)=\left[\begin{array}{ll}\overline{\tilde{C}}_{11}(t) & \overline{\tilde{C}}_{12}(t) \\ \tilde{\tilde{C}}_{21}(t) & \tilde{C}_{22}(t)\end{array}\right]$ and $\tilde{\varphi}(t)=\left[\begin{array}{l}\tilde{\varphi}_{1}(t) \\ \tilde{\varphi}_{2}(t)\end{array}\right]$ have appropriate dimensions. Now, $\tilde{\mathbf{S}}$ can be represented as two interconnected subsystems

$$
\begin{aligned}
\tilde{\mathbf{S}}_{1}: \dot{\tilde{x}}_{1}(t) & =\overline{\tilde{A}}_{11}(t) \tilde{x}_{1}(t)+\overline{\tilde{B}}_{11}(t) \tilde{u}_{1}(t)+\overline{\tilde{C}}_{11}(t) \tilde{x}_{1}(t-d) \\
& +\overline{\tilde{C}}_{12}(t) \tilde{x}_{2}(t-d)+\overline{\tilde{A}}_{12}(t) \tilde{x}_{2}(t)+\overline{\tilde{B}}_{12}(t) \tilde{u}_{2}(t), \\
\tilde{x}_{1}(t) & =\tilde{\varphi}_{1}(t), \quad-d \leq t \leq 0 \\
\tilde{\mathbf{S}}_{2}: \dot{\tilde{x}}_{2}(t) & =\overline{\tilde{A}}_{22}(t) \tilde{x}_{2}(t)+\overline{\tilde{B}}_{22}(t) \tilde{u}_{2}(t)+\overline{\tilde{C}}_{22}(t) \tilde{x}_{2}(t-d) \\
& +\overline{\tilde{C}}_{21}(t) \tilde{x}_{1}(t-d)+\overline{\tilde{A}}_{21}(t) \tilde{x}_{1}(t)+\overline{\tilde{B}}_{21}(t) \tilde{u}_{1}(t), \\
\tilde{x}_{2}(t) & =\tilde{\varphi}_{2}(t), \quad-d \leq t \leq 0,
\end{aligned}
$$

where $\overline{\tilde{A}}_{i i}(t), \overline{\tilde{B}}_{i i}(t), \overline{\tilde{C}}_{i i}(t), i=1,2$, are the matrices corresponding to the following decoupled subsystems:

$$
\begin{aligned}
\tilde{\mathbf{S}}_{\mathbf{D}}^{1}: & \dot{\tilde{x}}_{1}(t)=\overline{\tilde{A}}_{11}(t) \tilde{x}_{1}(t)+\overline{\tilde{B}}_{11}(t) \tilde{u}_{1}(t)+\overline{\tilde{C}}_{11}(t) \tilde{x}_{1}(t-d), \\
\tilde{x}_{1}(t) & =\tilde{\varphi}_{1}(t), \quad-d \leq t \leq 0, \\
\tilde{\mathbf{S}}_{\mathbf{D}}^{2}: & \dot{\tilde{x}}_{2}(t)=\overline{\tilde{A}}_{22}(t) \tilde{x}_{2}(t)+\overline{\tilde{B}}_{22}(t) \tilde{u}_{2}(t)+\overline{\tilde{C}}_{22}(t) \tilde{x}_{2}(t-d), \\
\tilde{x}_{2}(t) & =\tilde{\varphi}_{2}(t), \quad-d \leq t \leq 0,
\end{aligned}
$$

that is, when $\overline{\tilde{A}}_{i j}(t), \overline{\tilde{B}}_{i j}(t), \overline{\tilde{C}}_{i j}(t), i, j=1,2, i \neq j$, are considered null interconnection matrices.

Consider the cost functions associated to the decoupled subsystems $\tilde{\mathbf{S}}_{\mathbf{D}}^{1}, \tilde{\mathbf{S}}_{\mathbf{D}}^{2}$ in the form

$$
\begin{aligned}
& \tilde{J}_{D}^{1}\left(\tilde{x}_{10}, u_{1}\right)=\int_{0}^{\infty}\left[\tilde{x}_{1}^{T}(t) \tilde{Q}_{1}^{*} \tilde{x}_{1}(t)+u_{1}^{T}(t) \tilde{R}_{1}^{*} u_{1}(t)\right] d t \\
& \tilde{J}_{D}^{2}\left(\tilde{x}_{20}, u_{2}\right)=\int_{0}^{\infty}\left[\tilde{x}_{2}^{T}(t) \tilde{Q}_{2}^{*} \tilde{x}_{2}(t)+u_{2}^{T}(t) \tilde{R}_{2}^{*} u_{2}(t)\right] d t
\end{aligned}
$$

where $\tilde{x}_{10}$ and $\tilde{x}_{20}$ are the initial states of $\tilde{\mathbf{S}}_{\mathbf{D}}^{1}$ and $\tilde{\mathbf{S}}_{\mathbf{D}}^{2}$, respectively, and $\tilde{Q}_{1}^{*}, \tilde{Q}_{2}^{*}, \tilde{R}_{1}^{*}, \tilde{R}_{2}^{*}$ are appropriate weighting matrices. The total cost function is given by

$$
\tilde{J}_{D}=\tilde{J}_{D}^{1}\left(\tilde{x}_{10}, u_{1}\right)+\tilde{J}_{D}^{2}\left(\tilde{x}_{20}, u_{2}\right)
$$

Now, we can design local guaranteed cost controllers for both decoupled expanded subsystems $\tilde{\mathbf{S}}_{\mathbf{D}}^{1}, \tilde{\mathbf{S}}_{\mathbf{D}}^{2}$ by using Theorem 2. Thus, for each subsystem $\tilde{\mathbf{S}}_{\mathbf{D}}^{1}, \tilde{\mathbf{S}}_{\mathbf{D}}^{2}$ the local gain matrices

$$
\tilde{K}_{1}=\tilde{Y}_{1} \tilde{X}_{1}^{-1}, \quad \tilde{K}_{2}=\tilde{Y}_{2} \tilde{X}_{2}^{-1},
$$

are computed, where $\tilde{Y}_{1}, \tilde{X}_{1}, \tilde{Y}_{2}$ and $\tilde{X}_{2}$ are the same type of matrices as given in Theorem 2 . Then, a diagonal gain matrix

$$
\tilde{K}_{D}=\left[\begin{array}{cc}
\tilde{K}_{1} & 0 \\
0 & \tilde{K}_{2}
\end{array}\right]=\left[\begin{array}{cc:cc}
\tilde{K}_{11} & \tilde{K}_{12} & 0 & 0 \\
\hdashline 0 & 0 & \tilde{K}_{23} & \tilde{K}_{24}
\end{array}\right]
$$

is obtained, which will be contracted to

$$
K_{t d}=\tilde{K}_{D} V=\left[\begin{array}{cc:c}
\tilde{K}_{11} & \tilde{K}_{12} & 0 \\
\hdashline 0 & \tilde{K}_{23} & \tilde{K}_{24}
\end{array}\right] \text {. }
$$

A guaranteed cost control law $u_{t d}(t)=K_{t d} x(t)$ is finally implemented in the system $\mathbf{S}$. The gain matrix $K_{t d}$ corresponds to a tridiagonal block structure.

Remark 14. By Theorem 2 it is possible to use an LMI approach to get directly a tridiagonal gain matrix $K_{t d}$ for the initial system $\mathbf{S}$ with the structure given in (33). In fact, by imposing structural constraints on the matrices $Y$ and $X$ in the form

$$
Y=\left[\begin{array}{ccc}
y_{11} & y_{12} & 0 \\
0 & y_{22} & y_{23}
\end{array}\right], \quad X=\left[\begin{array}{ccc}
x_{11} & 0 & 0 \\
0 & x_{22} & 0 \\
0 & 0 & x_{33}
\end{array}\right],
$$

a tridiagonal gain matrix

$$
K_{t d}=Y X^{-1}=\left[\begin{array}{cc:c}
\tilde{K}_{11} & \tilde{K}_{12} & 0 \\
\hdashline 0 & \tilde{K}_{23} & \tilde{K}_{24}
\end{array}\right]
$$

for the system $\mathbf{S}$ is obtained, Šiljak and Zečević [2005]. However, with these structural restrictions on the matrices $Y$ and $X$ the possibility to obtain a reduced cost bound $J^{*}$ decreases considerably. Nevertheless, this difficulty can be overcome expanding the initial system, designing local gain matrices $\tilde{K}_{1}, \tilde{K}_{2}$ without any structural restrictions on the matrices $\tilde{Y}_{1}, \tilde{X}_{1}, \tilde{Y}_{2}, \tilde{X}_{2}$ given in (31) and, finally, contracting the diagonal gain matrix $\widetilde{K}_{D}$ to obtain a desired overlapping guaranteed cost control for the original system $\mathbf{S}$.

Moreover, it is well-known that from the standpoint of largescale applications, the computational complexity is a major concern. Thus, the approach presented in this paper allows us to work with subsystems instead of the whole given system.

\section{DESIGN PROCEDURE}

Consider guaranteed cost problems (1), (6) and (2), (7) satisfying (3), (4) and (5). We can observe that the cost bound $\tilde{J}^{*}$ in (15) depends on the matrices $\tilde{A}, \Delta \tilde{A}, \tilde{B}, \Delta \tilde{B}, \tilde{C}, \Delta \tilde{C}, \tilde{Q}^{*}$ and $\tilde{R}^{*}$. Consequently, for a given transformation $V$, the cost bound depends on the complementary matrices $M, N, M_{d}, M_{Q^{*}}$ and $N_{R^{*}}$. Then, we know the structure and the conditions on the complementary matrices $M, N, M_{d}, M_{Q^{*}}$ and $N_{R^{*}}$ which are given by relations (17) and Theorem 11 , but it is necessary to select their numerical values.

Let us consider the following steps: 
(1) Let $\left(A, B, C, E, E_{1}, E_{2}, E_{3}, Q^{*}, R^{*}\right)$ be the set of matrices which defines a system $\mathbf{S}$ together with an associated quadratic cost function $J\left(x_{0}, u\right)$. Consider also a continuous vector valued initial function $\varphi(t)$ and a time-delay $d$.

(2) Select complementary matrices $M$ and $N$ with the structures given in (24) or (25). These matrices have to satisfy the inclusion principle and must be chosen conveniently in such a way that the expanded subsystems are totally decoupled or weakly coupled.

(3) Choose the matrices $M_{R^{*}}, N_{R^{*}}$ to construct and expanded cost function as given in (7) where $\tilde{Q}^{*}=\operatorname{diag}\left\{\tilde{Q}_{1}^{*}, \tilde{Q}_{2}^{*}\right\}$ and $\tilde{R}^{*}=\operatorname{diag}\left\{\tilde{R}_{1}^{*}, \tilde{R}_{2}^{*}\right\}$. The decoupled expanded cost functions $\tilde{J}_{D}^{1}\left(\tilde{x}_{10}, u_{1}\right)$ and $\tilde{J}_{D}^{2}\left(\tilde{x}_{20}, u_{2}\right)$ are obtained.

(4) Design decentralized gain matrices $\tilde{K}_{1}, \tilde{K}_{2}$ for each decoupled subsystem by using the LMI approach. A diagonal gain matrix $\tilde{K}_{D}$ for the expanded space is obtained.

(5) Contract the diagonal gain matrix $\tilde{K}_{D}$ obtained in the previous step to the original system. A new tridiagonal gain matrix $K_{t d}$ is obtained, which serves as guaranteed cost controller for the initial system.

\section{NUMERICAL EXAMPLE}

Consider the system $\mathbf{S}$ given in (1) with an associated cost function (6) defined by the following matrices:

$$
\begin{gathered}
A=\left[\begin{array}{cc:c}
-1 & 1 & 0 \\
2 & -3 & -1 \\
\hdashline 0 & 0 & -1
\end{array}\right], B=\left[\begin{array}{ll}
1 & 0 \\
1 & 0 \\
0 & 1
\end{array}\right], C=\left[\begin{array}{cc:c}
0.1 & 0 & 0 \\
0 & 0 & 0 \\
\hdashline 0.1 & 0 & 0.1
\end{array}\right], \\
E=\left[\begin{array}{cc}
0.1 & 0 \\
0.1 & 0 \\
0 & 0.1
\end{array}\right], \quad E_{1}=E_{3}=\left[\begin{array}{ccc}
0.1 & 0 & 0 \\
0 & 0 & 0.1
\end{array}\right], \quad E_{2}=\left[\begin{array}{cc}
0.1 & 0.1 \\
0 & 0.1
\end{array}\right], \\
Q^{*}=\left[\begin{array}{lll}
1 & 0 & 0 \\
0 & 2 & 0 \\
0 & 0 & 1
\end{array}\right], \quad R^{*}=\left[\begin{array}{ll}
1 & 0 \\
0 & 1
\end{array}\right], \quad \varphi(t)=\left[\begin{array}{l}
t \\
1 \\
t
\end{array}\right], \quad d=1 .
\end{gathered}
$$

By choosing the complementary matrices

$$
M_{Q^{*}}=\left[\begin{array}{cccc}
0 & 0 & 0 & 0 \\
0 & 0.5 & -0.5 & 0 \\
0 & -0.5 & 0.5 & 0 \\
0 & 0 & 0 & 0
\end{array}\right], \quad N_{R^{*}}=\left[\begin{array}{ll}
0 & 0 \\
0 & 0
\end{array}\right],
$$

we obtain $\tilde{Q}^{*}=\operatorname{diag}\left\{I_{2}, I_{2}\right\}$ and $\tilde{R}^{*}=\operatorname{diag}\{1,1\}$. We consider that the complementary matrix $M_{d}$ given in (17) is a null matrix.

\subsection{Decentralized Control Design}

In this example, we expand the system $\mathbf{S}$ as an aggregation. Thus, we select the initial complementary matrices $M$ and $N$ in the form

$$
M=\left[\begin{array}{rrrr}
0 & 0 & 0 & 0 \\
2 & -1.5 & 1.5 & 1 \\
-2 & 1.5 & -1.5 & -1 \\
0 & 0 & 0 & 0
\end{array}\right], \quad N=\left[\begin{array}{rr}
0 & 0 \\
1 & 0 \\
-1 & 0 \\
0 & 0
\end{array}\right],
$$

according to (25). With this choice, the expanded matrices are the following:

$$
\begin{aligned}
& \tilde{A}=V A U+M=\left[\begin{array}{rrrr}
-1 & 0.5 & 0.5 & 0 \\
4 & -3 & 0 & 0 \\
0 & 0 & -3 & -2 \\
0 & 0 & 0 & -1
\end{array}\right], \\
& \tilde{B}=V B+N=\left[\begin{array}{ll}
1 & 0 \\
2 & 0 \\
0 & 0 \\
0 & 1
\end{array}\right] .
\end{aligned}
$$

We can observe that by adding these convenient complementary matrices $M$ and $N$, the matrices

$$
\begin{aligned}
& \overline{\tilde{A}}_{12}=\left[\begin{array}{cc}
0.5 & 0 \\
0 & 0
\end{array}\right], \quad \overline{\tilde{A}}_{21}=\left[\begin{array}{ll}
0 & 0 \\
0 & 0
\end{array}\right], \quad \overline{\tilde{B}}_{12}=\left[\begin{array}{l}
0 \\
0
\end{array}\right], \quad \overline{\tilde{B}}_{21}=\left[\begin{array}{l}
0 \\
0
\end{array}\right], \\
& \overline{\tilde{C}}_{12}=\left[\begin{array}{ll}
0 & 0 \\
0 & 0
\end{array}\right], \quad \overline{\tilde{C}}_{21}=\left[\begin{array}{cc}
0 & 0 \\
0.1 & 0
\end{array}\right]
\end{aligned}
$$

correspond to interconnection matrices with zero or small numerical values. Thus, the expanded space can almost be considered as a decoupled system. The diagonal gain matrix given in (32) is computed as

$$
\tilde{K}_{D}=\left[\begin{array}{cc}
\tilde{K}_{11} & 0 \\
0 & \tilde{K}_{22}
\end{array}\right]=\left[\begin{array}{cccc}
-1.8990 & -0.6390 & 0 & 0 \\
0 & 0 & 0.1401 & -1.4100
\end{array}\right]
$$

and the contracted tridiagonal gain matrix, denoted by $K_{t d}$ in (33), is given by

$$
K_{t d}=\tilde{K}_{D} V=\left[\begin{array}{ccc}
-1.8990 & -0.6390 & 0 \\
0 & 0.1401 & -1.4100
\end{array}\right] \text {, }
$$

which is used in the initial system $\mathbf{S}$ in order to control it. The corresponding bounded cost is $J^{*}=8.72$.

\subsection{Centralized Case}

We are interested in comparing the previous cost bound obtained following the procedure offered in the paper, with the cost bound corresponding to the diagonal gain matrix which can be calculated directly in the original system. In the centralized case, i.e. without any expansion-decoupling-contraction process, and by applying Theorem 2 , we have

$$
K_{t d}=Y X^{-1}=\left[\begin{array}{crc}
-1.6537 & -0.6756 & 0 \\
0 & 0.3960 & -1.1643
\end{array}\right] \text {. }
$$

Here, the bounded cost value is $J^{*}=23.02$. Thus, in the centralized case, the cost bound is greater than $J^{*}=8.72$. In fact, the increase in the centralized cost bound can be explained by the structural constraints imposed on the matrices $Y$ and $X$ given in (34).

\section{CONCLUSION}

This paper has dealt with guaranteed cost control for a class of linear continuous-time state-delay uncertain systems which are decomposed into overlapped subsystems. A design strategy to obtain a tridiagonal guaranteed cost controller for a given system has been presented. Complementary matrices which appear in a mathematical framework called the inclusion principle have been used. By means of this new strategy, both the cost bound of the performance index together with a computational complexity have been reduced significantly. The presented approach may help to exploit the potential of overlapping decomposition in practical applications mainly when the systems have large dimensions. A simple illustrative numerical example has been provided.

\section{REFERENCES}

L. Bakule and J. Rodellar. Decentralized control and overlapping decomposition of mechanical systems. part 1: System decomposition. part 2: Decentralized stabilization. International Journal of Control, 61(3):559-587, 1995.

L. Bakule, J. Rodellar, and J.M. Rossell. Structure of expansion-contraction matrices in the inclusion principle for dynamic systems. SIAM Journal on Matrix Analysis and Applications, 21(4):1136-1155, 2000a. 
L. Bakule, J. Rodellar, and J.M. Rossell. Generalized selection of complementary matrices in the inclusion principle. IEEE Transactions on Automatic Control, 45(6):1237-1243, $2000 \mathrm{~b}$.

L. Bakule, J. Rodellar, and J.M. Rossell. Controllabilityobservability of expanded composite systems. Linear Algebra and its Applications, 332-334:381-400, $2001 \mathrm{a}$.

L. Bakule, J. Rodellar, J.M. Rossell, and P. Rubió. Preservation of controllability-observability in expanded systems. IEEE Transactions on Automatic Control, 46(7):1155-1162, 2001b.

L. Bakule, J. Rodellar, and J.M. Rossell. Overlapping quadratic optimal control of linear time-varying commutative systems. SIAM Journal on Control and Optimization, 40(5):16111627, 2002a.

L. Bakule, J. Rodellar, and J.M. Rossell. Overlapping guaranteed cost control for time-varying discrete-time uncertain systems. In Proceedings of the American Control Conference, pages 1705-1710, Anchorage, Alaska, USA, $2002 \mathrm{~b}$.

L. Bakule, F. Paulet-Crainiceanu, J. Rodellar, and J.M. Rossell. Overlapping reliable control for a cable-stayed bridge benchmark. IEEE Transactions on Control Systems Technology, 13 (4):663-669, 2005a.

L. Bakule, J. Rodellar, and J.M. Rossell. Overlapping guaranteed cost control for uncertain continuous-time delayed systems. In Proceedings of the 16th IFAC World Congress, Prague, Czech Republic, 2005b.

L. Bakule, J. Rodellar, and J.M. Rossell. Overlapping resilient $H_{\infty}$ control for uncertain time-delayed systems. In Proceedings of the 44th IEEE Conference on Decision and Control and European Control Conference (CDC-ECC'05), pages 2290-2295, Sevilla, Spain, 2005c.

M. Ikeda and D.D. Šiljak. Overlapping decentralized control with input, state, and output inclusion. Control-Theory and Advanced Technology, 2(2):155-172, 1986.

M. Ikeda and D.D. Šiljak. Overlapping decompositions, expansions and contractions of dynamic systems. Large Scale Systems, 1(1):29-38, 1980.

M. Ikeda, D.D. Šiljak, and D.E. White. Decentralized control with overlapping information sets. Journal of Optimization Theory and Applications, 34(2):279-310, 1981.

M. Ikeda, D.D. Śiljak, and D.E. White. An inclusion principle for dynamic systems. IEEE Transactions on Automatic Control, 29(3):244-249, 1984a.

M. Ikeda, D.D. Šiljak, and D.E. White. Overlapping decentralized control of linear time-varying systems. In Advances in Large Scale Systems, volume 1, pages 93-116. JAI Press, Greenwich, Connecticut, 1984b.

X. Li, X. Chen, and Ch. Wang. $H_{\infty}$ control of overlapping interconnected systems. In Proceedings of the 3rd. World Congress on Intelligent Control and Automation, pages 3368-3371, Hefei, P.R. China, 2000.

H. Mukaidani. An LMI approach to guaranteed cost control for uncertain delay systems. IEEE Transactions on Circuits and Systems-I: Fundamental Theory and Applications, 50 (6):795-800, 2003.

M.E. Sezer and D.D. Šiljak. Nested epsilon decomposition of linear systems: weakly coupled and overlapping blocks. SIAM Journal of Matrix Analysis and Its Applications, 12 (3):521-533, 1991.

S.S. Stanković, X.-B. Chen, and D.D. Šiljak. Stochastic inclusion principle applied to decentralized automatic generation control. International Journal of Control, 72(3):276-288,
1999.

S.S. Stanković, M. Stanojević, and D.D. Šiljak. Decentralized overlapping control of a platoon of vehicles. IEEE Transactions on Control Systems Technology, 8(5):816-832, 2000.

D.M. Stipanović, G. Inalhan, R. Teo, and C.J. Tomlin. Decentralized overlapping control of a formation of unmanned aerial vehicles. Automatica, 40:1285-1296, 2004.

D.D. Šiljak. Decentralized Control of Complex Systems. Academic Press, New York, USA, 1991.

D.D. Śiljak and A.I. Zečević. Control of large-scale systems: Beyond decentralized feedback. Annual Reviews in Control, 29:169-179, 2005. 